\title{
Spatial frequency analysis of block letters does not predict experimental confusions
}

\author{
STEPHEN COFFIN \\ Universitv of Michigan, Ann Arbor, Michigan 48104
}

\begin{abstract}
To test the generality of spatial frequency channels models of vision, matrices of similarities between spatial frequency compositions of letters of the alphabet were generated mathematically. Methods and assumptions were chosen to reflect probable neural underpinnings of spatial frequency analysis mechanisms. These similarity matrices were tested against experimentally produced confusion matrices. The similarity matrices predicted subjects' confusions poorly: the best predictions were made, not by any of the spatial frequency similarities, but by similarities between the original letter stimuli input to the spatial frequency analysis programs. Previously reported feature analy tic models seem to better describe the confusion patterns.
\end{abstract}

Many investigators have attempted to analyze the behavior of the human visual system in terms of spatial frequency channels, which they feel can account for subjects' behavior in contrast grating experiments. These theories compete with older feature analytic approaches to perception. However, attempts to compare the spatial frequency analysis model with subjects' performance have been primarily based on techniques and assumptions which maximize the performance of the model rather than upon those assumptions which reflect the capabilities of the subject.

The typical spatial frequency channel model for vision (Graham, 1976; Sachs, Nachmias, \& Robson, 1971; Thomas, 1970) postulates elongated receptive fields with off-on-off (or on-off-on) organization, of several different widths. Receptive fields of similar size pool their responses to a stimulus, leading to size-tuned channels which respond, not to specific lines or features of a stimulus at a specific retinal location, but rather to a given spatial frequency, which corresponds to the width of the receptive fields making up the pool. According to these theories, response of many size-tuned channels comprise the neural response to any stimulus, and the output of these channels determines the subjects perception of the stimulus presented. In many cases, sensitivity to Fourier components of the stimulus has been analyzed, and used as a measure of the sensitivity of the neural spatial frequency channels (Graham \& Nachmias, 1971; Quick \& Reichert, 1975; Henning, Hertz, \& Broadbent, 1975). In effect, it has been proposed that the visual system is performing a Fourier analysis of stimuli, or more accurately, is

Supported in part by NIMH Posidocioral Fellowship 5 F22 MH00837, and by NEI Vision Research Training Grant 1 T32 EY07022-03. Thanks are extended to Dr. D. H. Krantz for his many helpful comments. filtering complex input into the responses of separate channels (Campbel! \& Robson, 1968; Maffei \& Fiorentini, 1973; Pollen, Taylor, \& Lee, 1972). Current theory is somewhat unclear about the fate of phase information following the spatial frequency analysis: it could be discarded in summation of offon-off units with on-off-on units, or could be maintained by separation of these types. Further, it is unclear whether response of all spatial frequency channels is equally utilized or if low spatial frequencies are relatively emphasized, or even whether the spatial frequency channels are of equal size throughout the spatial frequency domain. Possibly, sensitivity to spatial frequency (Campbell \& Robson, 1968) weights the contribution of the frequency bands, or bands are shaped into octave-wide regions (Stromeyer \& Julesz, 1972).

In this narrow-band channels model, recognition may consist primarily of a template correlation of the Fourier representation of the visual input with stored Fourier representations of known patterns. The highest correlation is chosen as a match, and the visual input is categorized as this pattern. That is, the more similar the Fourier representations of two patterns, the more likely they are to be confused.

The other major approach proposes that stimuli are analyzed on the basis of edge and feature detection. The receptive fields themselves are the building blocks of perception, and their response, while similar in form to that mentioned above, is pooled into lines and edges, which, along with position information presumably coded as different sites on the cortical representation of the retina, make up the features of the visual world (Gibson, 1969; Neisser, 1967; Selfridge, 1959). Mathematically, the feature model may be represented by broadly tuned channels with either even (center-surround receptive fields) or odd (elongated receptive fields) symmetry. of course, representation of position information 
is quite different in the two approaches, with phase coding in the Fourier model, and channel/spatial position coding necessary in the feature model.

These two models make quite different predictions concerning the perception of real-world stimuli such as letters of the alphabet. Stimulus confusion should be based on spatial frequency composition if the channels model is correct, but on presence and position of edges, angles, curves, etc., if the features model is correct. While it is apparent that some similarities in the spatial frequency composition of stimuli which have similar feature characteristics will appear, there should be sufficient different between the two analyses to allow discriminative testing of the theories.

To this end, a computer program to determine spatial frequency composition of two-dimensional stimuli was written, and applied to the letters of the alphabet. It is predicted that greater similarity between spectra would be associated with greater confusability between the original stimuli in human observers if the spatial frequency model was correct. Otherwise, similarities between the spectra would be poorly related to subjects' confusions.

\section{THE SIMULATION PROGRAM}

The program, written in Fortran, calculates a twodimensional fast Fourier transform (Webb, 1970) of its input array, a 128-point square matrix on which any stimulus may be drawn. Since, apparently, the vertical and horizontal spatial frequency components are detected independently in vision (Carlson, Cohen, \& Gorog, 1977), this two-dimensional mathematical procedure will be a satisfactory representation of two-dimensional visual performance. The in-place fast Fourier transform of the $128 \times 128$ point "stimulus" yielded a $128 \times 128$ point spatial frequency representation. The 128-point "retina" dimension was chosen to represent as nearly as possible the visual capabilities: if the "retina" is assumed to be about $2.56^{\circ}$ of visual angle square, then the frequency resolution of the simulation is about .39 cycles $/ \mathrm{deg}$, which is about the low spatial frequency resolution capacity of the human visual system. The Nyquist "folding" frequency is about 25 cycles/deg, near human high-frequency resolution limits (Campbell \& Robson, 1968; Quick \& Reichert, 1975). While the frequency resolution in the middle of the frequency range is better than the width of narrow-band channels in the visual system, this narrow bandwidth leads to greater sensitivity in the model than in the visual system. In effect, the model can be better than the performance of the visual system. In addition, summation of adjacent frequency channels in the simulation in various ways can better imitate human spatial frequency response.
Since it is not clear whether phase differences are maintained in vision, two versions of each spectrum were calculated. In one version, each stimulus was represented by both its sine and cosine transform (called raw spectra below). In the second, these were combined into a single amplitude term at each frequency by squared-modulus summation of the sine and cosine terms at that frequency. This, in effect, assumes constant phase relations between the various spatial frequency components which are extracted from the input stimulus. Performance of these amplitude spectra were compared with the raw spectra in predicting experimental confusions.

\section{STIMULI TO THE SIMULATION}

The present experiments analyzed large block letters with positive contrast, or bright letters on a dark background. An example of the stimulus set is shown on the inset of Figure 1. All letters were large block capitals which filled almost the entire $128 \times$ 128 point matrix. Because of the large stroke width of the stimuli and the fact that the stimulus form filled almost the entire "retina," primarily low frequencies were seen in the spectra. Amplitude spectra of all 26 letters were similar to that shown for the letter " $A$ " in Figure 1, with the majority of the energy in the spectra at low frequencies, and only small high-frequency edge-related components.

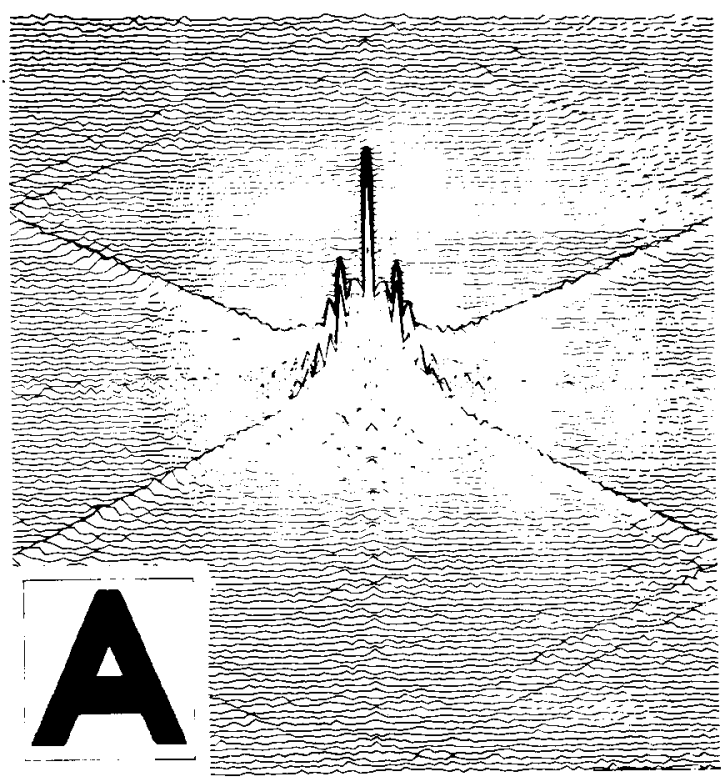

Figure 1. Two-dimensional Fourier spectrum squared-modulus summation of real and imaginary components at each frequency bin for the letter " $A$ " (inset). Direct current power is at the center, and increased spatial frequency in the vertical dimension is expressed as vertical distance from the center. Increased spatial frequency in the horizontal dimension is expressed as horizontal distance from the center. Height of the peak is the amplitude of that spatial frequency component. Most energy is at the low frequencies. This amplitude spectrum is radially symmetric. 


\section{SPECTRAL AVERAGING}

Along with computation of the raw Fourier transforms and the complete squared modulus amplitude spectra, several other combinations of frequency bins in the spectra were calculated. First, for both raw and amplitude spectra for each letter, various lowfrequency portions were extracted. These lowfrequency spectra were approximately 0-1.25, 0-2.50, $0-5.0$, and $0-10.0$ cycles $/ \mathrm{deg}$. These were matrices of dimension $8 \times 8,12 \times 12,26 \times 26$, and $52 \times 52$ frequency bins, respectively, centered at the dc point in the spectra. These were examined, since several reports suggest that primarily low frequencies are used by subjects in these experiments (Goble, 1975; Granlund, 1972; Kabrisky, Tallman, Day, \& Radoy, 1970). In addition, amplitudes in octave-wide spatial frequency bands along both horizontal and vertical dimensions were summed together (after Stromeyer \& Julesz, 1972) to yield widths of approximately $0.0, .4, .8-1.2,1.5-2.5,2.8-5.0,5.4-10.0$, and 10.4$20.0 \mathrm{cycles} / \mathrm{deg}$. That is, for the vertical dimension, and separately for the horizontal dimension, all points within blocks $0,1,2-4,5-8,9-16$, and 17-32 frequency bins away from the dc point were averaged. Thus, along a $45^{\circ}$ oblique from the dc point, bins of size $1,1,3 \times 3,4 \times 4,8 \times 8$, and $16 \times 16$ points were obtained, resulting in one $11 \times 11$ point spectrum. This procedure is justified if octave-wide bands can be obtained by stimulation at any orienta- tion, and if detection of gratings in different orientations is independent (Carlson et al., 1977). Finally, these octave-wide bands were shaped by human spatial frequency sensitivity as measured by Campbell and Robson (1968), so that each bin was weighted by human response to that frequency range. Consequently, in this version, both high- and lowfrequency bins were weighted less heavily than bins in the middle of the frequency range. All these manipulations were performed both on the raw spectra and the amplitude spectra, for a total of 14 sets of calculated spectra. In addition, the original letters (which were input to the spectral analysis program) were subjected to the same analyses as the spectra.

\section{SIMILARITIES BETWEEN SPECTRA}

Similarity between any two spectra within a calculation process was assessed by calculating the linear correlation coefficient between corresponding frequencies or frequency bins.

Correlation coefficients between all pairs of spectra, for two representative sets of spectra, are shown in Table 1. Most of the other calculated spectra yielded relatively similar results. Many of these correlations were high, as expected, because of the similarity of the letters used in the calculation of the spectra. For example, "E"' and " $F$ " show high correlation (.87 and .97), as do "G" and "Q" (.96

Table 1

Similarities Between Spectra

\begin{tabular}{|c|c|c|c|c|c|c|c|c|c|c|c|c|c|c|c|c|c|c|c|c|c|c|c|c|c|c|}
\hline & $\mathbf{A}$ & B & $\mathrm{C}$ & D & $\mathrm{E}$ & $F$ & $\mathbf{G}$ & $\mathbf{H}$ & I & $\mathbf{J}$ & $\mathbf{K}$ & $\mathbf{L}$ & $\mathbf{M}$ & $\mathbf{N}$ & 0 & $\mathbf{P}$ & $Q$ & $\mathbf{R}$ & $S$ & $\mathrm{~T}$ & $\mathbf{U}$ & V & W & $X$ & $\mathbf{Y}$ & Z \\
\hline A & 0 & 64 & 48 & 22 & 44 & 60 & 52 & 30 & 87 & 57 & 89 & 4 & 35 & 15 & 31 & 75 & 49 & 76 & 81 & 69 & -49 & 72 & 68 & 73 & 83 & 26 \\
\hline B & 81 & 0 & 68 & 73 & 43 & 50 & 64 & 78 & 51 & 10 & 70 & 47 & 58 & 46 & 76 & 87 & 75 & 91 & 87 & & -7 & 75 & 90 & 32 & 64 & -5 \\
\hline C & 89 & 89 & 0 & 82 & 80 & 67 & 86 & 64 & 40 & 20 & 60 & 72 & 62 & 62 & 80 & 68 & 82 & 77 & 68 & 50 & 31 & 54 & 69 & 38 & 42 & 42 \\
\hline D & 84 & 96 & 91 & 0 & 68 & 50 & 71 & 83 & 17 & 4 & 43 & 78 & 60 & 64 & 78 & 61 & 75 & 72 & 52 & 32 & 47 & 50 & 71 & 24 & 29 & 30 \\
\hline E & 84 & 96 & 90 & 93 & 0 & 87 & 64 & 55 & 36 & 29 & 56 & 71 & 52 & 50 & 44 & 62 & 51 & 62 & 37 & 54 & 29 & 36 & 49 & 52 & 33 & 64 \\
\hline $\mathrm{F}$ & 82 & 96 & 89 & 93 & 97 & 0 & 41 & 45 & 44 & 21 & 66 & 62 & 37 & 26 & 27 & 82 & 32 & 68 & 40 & 44 & -10 & 35 & 43 & 49 & 40 & 37 \\
\hline G & 87 & 91 & 95 & 92 & 90 & 89 & 0 & 69 & 40 & 35 & 54 & 39 & 82 & 81 & 88 & 51 & 96 & 75 & 76 & 53 & 42 & 61 & 78 & 43 & 45 & 49 \\
\hline H & 76 & 92 & 84 & 93 & 92 & 92 & 86 & 0 & 5 & -9 & 34 & 51 & 78 & 76 & 83 & 66 & 78 & 79 & 58 & 8 & 41 & 41 & 74 & 8 & 22 & 6 \\
\hline I & 83 & 84 & 81 & 82 & 88 & 86 & 78 & 77 & 0 & 77 & 93 & 7 & 13 & -4 & 14 & 52 & 37 & 55 & 73 & 89 & -48 & 83 & 64 & 88 & 95 & 41 \\
\hline $\mathbf{J}$ & 90 & 89 & 92 & 91 & 91 & 90 & 89 & 82 & 90 & 0 & 61 & -18 & 19 & 7 & 0 & 9 & 26 & 20 & 43 & 88 & -11 & 65 & 42 & 88 & 71 & 75 \\
\hline $\mathbf{K}$ & 87 & 91 & 89 & 92 & 90 & 90 & 89 & 89 & 89 & 92 & 0 & 36 & 29 & 14 & 33 & 75 & 53 & 78 & 82 & 83 & -36 & 85 & 77 & 85 & 93 & 36 \\
\hline L & 81 & 91 & 85 & 92 & 92 & 92 & 84 & 92 & 88 & 89 & 94 & 0 & 26 & 33 & 43 & 53 & 38 & 49 & 23 & 20 & 33 & 24 & 36 & 15 & 14 & 18 \\
\hline $\mathbf{M}$ & 86 & 89 & 85 & 91 & 90 & 89 & 88 & 93 & 81 & 85 & 90 & 88 & 0 & 96 & 81 & 47 & 80 & 63 & 59 & 24 & 56 & 49 & 71 & 23 & 26 & 37 \\
\hline $\mathbf{N}$ & 85 & 89 & 86 & 92 & 90 & 88 & 88 & 91 & 80 & 87 & 91 & 87 & 97 & 0 & 82 & 33 & 79 & 53 & 46 & 14 & 73 & 35 & 60 & 11 & 9 & 36 \\
\hline 0 & 84 & 87 & 94 & 91 & 86 & 95 & 94 & 83 & 71 & 86 & 84 & 80 & 84 & 86 & 0 & 54 & 94 & 73 & 72 & 17 & 47 & 49 & 76 & 6 & 26 & 12 \\
\hline $\mathbf{P}$ & 85 & 97 & 92 & 96 & 96 & 97 & 91 & 94 & 85 & 91 & 92 & 94 & 92 & 90 & 88 & 0 & 55 & 91 & 72 & 36 & -25 & 58 & 71 & 39 & 57 & 1 \\
\hline$Q$ & 86 & 91 & 94 & 93 & 88 & 88 & 96 & 86 & 75 & 88 & 89 & 85 & 87 & 89 & 96 & 91 & 0 & 80 & 83 & 43 & 38 & 64 & 85 & 34 & 46 & 30 \\
\hline $\mathbf{R}$ & 82 & 98 & 89 & 96 & 94 & 94 & 90 & 94 & 81 & 89 & 91 & 92 & 90 & 89 & 86 & 98 & 90 & 0 & 87 & 46 & -6 & 68 & 86 & 47 & 63 & 12 \\
\hline$S$ & 84 & 91 & 92 & 86 & 90 & 90 & 92 & 81 & 83 & 88 & 87 & 84 & 81 & 82 & 87 & 90 & 90 & 89 & 0 & 58 & -13 & 85 & 92 & 56 & 81 & 14 \\
\hline $\mathrm{T}$ & 82 & 89 & 85 & 88 & 93 & 92 & 84 & 89 & 93 & 90 & 92 & 91 & 89 & 89 & 77 & 90 & 81 & 87 & 84 & 0 & -12 & 79 & 60 & 95 & 84 & 75 \\
\hline $\mathbf{U}$ & 82 & 91 & 86 & 92 & 92 & 93 & 89 & 93 & 82 & 87 & 90 & 91 & 94 & 94 & 88 & 92 & 91 & 89 & 85 & 91 & 0 & -9 & 10 & -19 & -36 & 38 \\
\hline V & 94 & 85 & 89 & 89 & 87 & 85 & 89 & 81 & 84 & 91 & 90 & 85 & 87 & 86 & 84 & 88 & 87 & 86 & 83 & 85 & 84 & 0 & 90 & 76 & 93 & 38 \\
\hline W & 87 & 88 & 87 & 93 & 89 & 87 & 90 & 91 & 75 & 84 & 87 & 85 & 94 & 92 & 87 & 91 & 90 & 90 & 81 & 83 & 88 & 89 & 0 & 57 & 78 & 27 \\
\hline $\mathbf{X}$ & 88 & 86 & 88 & 87 & 83 & 83 & 90 & 77 & 81 & 89 & 92 & 83 & 81 & 84 & 87 & 85 & 90 & 84 & 87 & 82 & 83 & 88 & 81 & 0 & 86 & 70 \\
\hline$Y$ & 92 & 80 & 86 & 80 & 82 & 82 & 86 & 73 & 87 & 90 & 89 & 81 & 82 & 82 & 80 & 82 & 84 & 79 & 85 & 86 & 82 & 90 & 78 & 91 & 0 & 33 \\
\hline Z & 87 & 90 & 88 & 89 & 93 & 89 & 89 & 85 & 87 & 89 & 88 & 87 & 89 & 86 & 84 & 91 & 86 & 88 & 87 & 88 & 87 & 85 & 88 & 84 & 83 & 0 \\
\hline
\end{tabular}

Note-Top and right is raw spectra, octave wide bands, shaped by sensitivity; bottom and left: squared modulus amplitude spectra, complete matrix. 
and .96). This results from the similar letter shape and similar spatial frequency composition. Conversely, similarity between spectra of some letters that are dissimilar in appearance was unusually low. For example, "O" and "I" (.14 and .71), and "I" and " $\mathrm{H}$ " (.05 and .77). However, some pairs show very different behavior with the different spectral averaging procedures; for example, " $D$ " and " $B$ " (.73 and .96) and "F" and " $U$ " ( -.10 and .93). These differences are the basis for testing the relative merits of the different spectral techniques in predicting experimental confusions. In general, it can be noted that the amplitude spectra produce greatly increased similarity between spectra.

\section{SPECTRAL SIMILARITY MATRICES} VS. EXPERIMENTAL CONFUSIONS

If these spectra can be used as estimates of the spatial frequency response of visual channels, then these similarities between spectra should predict confusions that subjects make between the letters. Similar responses of neural channels should lead to similar reports by subjects. Since many experimentally determined confusion matrices between letters have been published (Fisher, Monty, \& Glucksburg, 1969; Hodge, 1962; Pew \& Gardner, cited in Fisher et al., 1969; Townsend, 1971; Gibson,
Osser, Schiff, \& Smith, Note 1), with varying experimental conditions, subject pools, and stimulus design, the spectral similarity matrices were related to these visual confusion matrices. If the pattern of the matrices is similar, then we have evidence in favor of the spatial frequency analysis model. These experiments were conducted under severely degraded stimulus conditions, as near threshold as could be managed. Thus, the nonlinearities inherent in suprathreshold stimulus presentation were reduced as much as possible, reducing possible nonlinearities in a spatial frequency representation.

Table 2 shows correlation coefficients calculated between the pairs of confusion matrices reported in the literature, as well as the spectral similarity matrices. Both linear (Pearson's $r$ ) and rank (Spearman's rho) correlations were calculated, and they agree well with each other. Because of the differing experimental conditions in many of the reports, the confusion matrices were associated with each other, to get an estimate of the replicability of the confusion matrix paradigm. In all cases, similarity between experimentally determined confusion matrices was significantly greater than chance (correlation of .14 is significant at $p=.01$ ). Many of these correlations were extremely high, accounting for as much as $77 \%$ of the variance. However, while some of the correlations between these experimental

Table 2

Correlations Between Spectral and Experimental Confusion Matrices

\begin{tabular}{|c|c|c|c|c|c|c|c|c|c|c|c|c|c|c|c|c|c|c|c|c|c|c|c|}
\hline & 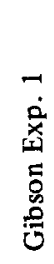 & 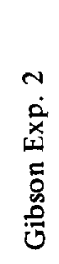 & 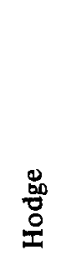 & 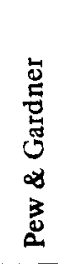 & 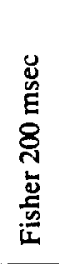 & 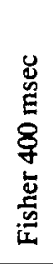 & 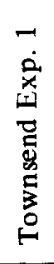 & 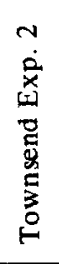 & 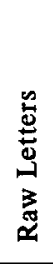 & 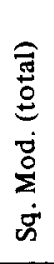 & 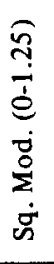 & $\begin{array}{l}\widetilde{n} \\
\tilde{y} \\
\dot{d} \\
\dot{8} \\
\dot{0} \\
\dot{\Sigma} \\
\dot{\delta}\end{array}$ & 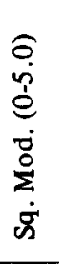 & $\begin{array}{l}\hat{\theta} \\
\dot{d} \\
\dot{e} \\
\dot{g} \\
\dot{0} \\
\dot{g}\end{array}$ & 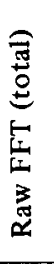 & 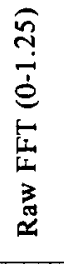 & 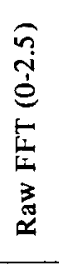 & 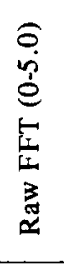 & 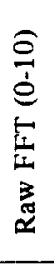 & 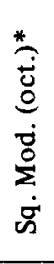 & 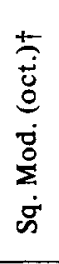 & 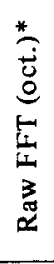 & 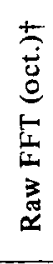 \\
\hline Gibson Exp. 1 & 0 & 64 & 41 & 56 & 38 & 34 & 48 & 41 & 42 & 36 & 33 & 37 & 36 & 37 & 38 & 35 & 36 & 37 & 37 & 25 & 31 & 22 & 22 \\
\hline Gibson Exp. 2 & 39 & 0 & 32 & 43 & 33 & 32 & 39 & 32 & 28 & 25 & 21 & 24 & 24 & 25 & 22 & 19 & 21 & 22 & 21 & 13 & 18 & 10 & 14 \\
\hline Hodge & 32 & 26 & 0 & 59 & 50 & 42 & 59 & 45 & 42 & 24 & 21 & 24 & 24 & 24 & 34 & 32 & 33 & 34 & 34 & 15 & 18 & 23 & 24 \\
\hline Pew \& Gardner & 40 & 26 & 34 & 0 & 56 & 48 & 60 & 62 & 42 & 30 & 26 & 28 & 30 & 31 & 31 & 27 & 28 & 30 & 30 & 15 & 24 & 21 & 19 \\
\hline Fisher $200 \mathrm{msec}$ & 27 & 24 & 42 & 48 & 0 & 88 & 74 & 53 & 42 & 27 & 20 & 23 & 26 & 26 & 29 & 27 & 28 & 28 & 28 & 13 & 22 & 21 & 24 \\
\hline Fisher $400 \mathrm{msec}$ & 28 & 29 & 34 & 46 & 60 & 0 & 64 & 46 & 39 & 26 & 18 & 22 & 24 & 25 & 26 & 23 & 25 & 26 & 26 & 8 & 20 & 18 & 22 \\
\hline Townsend Exp. 1 & 36 & 25 & 42 & 42 & 50 & 36 & 0 & 63 & 48 & 34 & 27 & 32 & 33 & 34 & 36 & 33 & 35 & 35 & 35 & 24 & 28 & 31 & 30 \\
\hline Townsend Exp. 2 & 23 & 15 & 22 & 36 & 30 & 28 & 44 & 0 & 26 & 11 & 7 & 10 & 10 & 10 & 14 & 12 & 13 & 13 & 13 & -1 & 6 & 21 & 18 \\
\hline Raw Letters & 36 & 24 & 44 & 34 & 40 & 40 & 41 & 14 & 0 & 61 & 56 & 61 & 62 & 61 & 87 & 84 & 86 & 87 & 86 & 43 & 51 & 45 & 50 \\
\hline Sq. Mod. (total) & 33 & 24 & 28 & 28 & 23 & 26 & 29 & 4 & 58 & 0 & 94 & 98 & 99 & 99 & 64 & 62 & 64 & 65 & 64 & 76 & 92 & 32 & 30 \\
\hline Sq. Mod. $(0-1.25)$ & 32 & 22 & 27 & 26 & 16 & 19 & 24 & 1 & 55 & 94 & 0 & 98 & 96 & 96 & 62 & 62 & 63 & 63 & 61 & 83 & 87 & 31 & 27 \\
\hline Sq. Mod. $(0-2.5)$ & 34 & 24 & 28 & 26 & 19 & 22 & 28 & 2 & 60 & 98 & 97 & 0 & 99 & 98 & 67 & 66 & 67 & 68 & 66 & 81 & 90 & 34 & 32 \\
\hline Sq. Mod. (0-5.0) & 33 & 24 & 28 & 27 & 22 & 24 & 29 & 2 & 60 & 98 & 96 & 99 & 0 & 99 & 66 & 64 & 66 & 67 & 65 & 78 & 92 & 34 & 32 \\
\hline Sq. Mod. $(0-10)$ & 34 & 25 & 28 & 28 & 23 & 25 & 30 & 3 & 58 & 99 & 96 & 98 & 99 & 0 & 65 & 63 & 65 & 66 & 64 & 78 & 92 & 33 & 31 \\
\hline Raw FFT (total) & 32 & 23 & 42 & 22 & 26 & 27 & 33 & 4 & 83 & 62 & 62 & 66 & 64 & 63 & 0 & 98 & 99 & 99 & 98 & 58 & 59 & 59 & 58 \\
\hline Raw FFT $(0.1 .25)$ & 33 & 22 & 44 & 21 & 28 & 27 & 34 & 4 & 83 & 62 & 62 & 66 & 64 & 63 & 98 & 0 & 99 & 99 & 97 & 62 & 59 & 60 & 58 \\
\hline Raw FFT $(0-2.5)$ & 33 & 23 & 43 & 20 & 27 & 28 & 34 & 4 & 84 & 64 & 64 & 68 & 66 & 65 & 98 & 100 & 0 & 100 & 98 & 61 & 60 & 60 & 59 \\
\hline Raw FFT $(0-5.0)$ & 33 & 22 & 41 & 21 & 26 & 28 & 32 & 4 & 84 & 64 & 63 & 67 & 66 & 64 & 98 & 99 & 100 & 0 & 99 & 60 & 60 & 60 & 58 \\
\hline Raw FFT $(0-10)$ & 32 & 22 & 41 & 20 & 26 & 27 & 31 & 3 & 82 & 61 & 61 & 65 & 64 & 62 & 97 & 97 & 98 & 98 & 0 & 58 & 58 & 59 & 58 \\
\hline Sq. Mod. (oct.)* & 28 & 13 & 23 & 10 & 10 & 6 & 26 & -8 & 43 & 74 & 77 & 77 & 76 & 76 & 60 & 62 & 62 & 62 & 60 & 0 & 78 & 33 & 31 \\
\hline Sq. Mod. (oct.) $†$ & 31 & 18 & 24 & 21 & 21 & 21 & 28 & 1 & 48 & 91 & 86 & 89 & 91 & 92 & 56 & 58 & 58 & 58 & 56 & 80 & 0 & 32 & 32 \\
\hline Raw FFT (oct.)* & 18 & 12 & 22 & 20 & 16 & 16 & 34 & 15 & 43 & 33 & 33 & 36 & 35 & 35 & 59 & 61 & 60 & 60 & 59 & 39 & 33 & 0 & 82 \\
\hline Raw FFT (oct.) $\dagger$ & 15 & 14 & 26 & 18 & 19 & 18 & 34 & 13 & 44 & 30 & 28 & 33 & 32 & 32 & 55 & 56 & 56 & 55 & 55 & 37 & 31 & 82 & 0 \\
\hline
\end{tabular}


confusion matrices and the spectral similarity matrices exceeded $p=.01$, these correlations were much smaller. In fact, in no case could as much as $16 \%$ of the variance be accounted for by the spatial frequency analysis. More importantly, in every case, similarity between the original letters predicted experimental confusion patterns better than any of the spectra. While our stimulus set was not exactly the same as any reported in these experimental tests, neither were most of the experimental stimulus sets especially similar to each other. Yet, relatively high replicability exists between these experiments, while the spectral similarity data seem divergent from all of them.

On the other hand, examination of the experimental confusion matrices shows that a feature model does fairly well in describing the pattern of confusions. Gibson's feature list accounted for up to $40 \%$ of the variance for various letters (Gibson et al., Note 1), and Künnapas' factor analysis of confusions between a partial list of letters revealed three main factors, which were interpreted as three features ("rectangularity," "roundness," and "vertical linearity"). The more of these features in common, the more confusable were the letters (Künnapas, 1966).

\section{DISCUSSION}

Several other attempts to use Fourier analysis of letters to predict subjects' confusions have appeared (Goble, 1975; Granlund, 1972; Kabrisky et al., 1970). These report better correlation between subjects' confusions and spectral similarity than the present attempt. However, these reports differ from the present approach in several fundamental ways. Kabrisky and Goble report that the low spatial frequency components of their spectra are far more diagnostic in predicting experimental confusions than are the higher spatial frequencies, while we find that the opposite is true: the more information retained in the spectra, the better the predictions of experimental confusability. Both of these reports, however, produced experimental confusion matrices in a "dotted" environment: Kabrisky presented letters formed of rows of dots, and Goble presented his letters in a dotted visual noise mask. The presence of so much irrelevant high spatial frequency information in these dotted patterns relatively emphasizes the discriminative value of the low-frequency information in the letter stimuli. When the test stimuli are presented with no visual noise mask, as are the experimental reports compared with the spectra here, both high and low spatial frequency information is useful in making discriminations, and leads to our result that the low frequencies do not do as well as the overall spectra. Secondly, these other reports (especially Granlund \& Goble) used complex "pattern recogni- tion" processors to generate the final spectral similarities, while we used only a direct template match (linear correlation between spectra) to generate our spectral similarity matrices. While the structure of the visual system may not rule out these a priori pre- and postprocessing steps, current knowledge of visual processing makes them unlikely. Preprocessing is probably limited to dissection of the input stimulus into the response of the receptive fields. In any case, manipulations of the spatial frequency analysis process should be based on knowledge of visual mechanisms if the model is to be viable.

Of course, several factors could confound the present results. First, the experimental confusion matrices could have been determined at suprathreshold levels, such that nonlinearities were significant in the spatial frequency representations. At significantly suprathreshold levels, however, subjects make virtually no errors in a simple letter identification task, and detection and recognition thresholds for letter stimuli are surprisingly close together. Second, if the spatial frequency channels are sufficiently broadly tuned for them to perform only very crude Fourier analysis, our narrow-band channel representation will fail. However, even the octave bandwidth spectra did not improve the fit of the spectra to the experimental data. Thirdly, the calculated spectra may contain nonlinearities which significantly reduce the fit to the experimental data. That nonlinearities exist in the spectra is apparent when the correlation between the raw letters and the raw spectra is examined. Since the Fourier transform is a linear transformation, it should produce a correlation of one with the untransformed stimulus set. Probable sources of the achieved correlation of .87 and .83 are failures of the Nyquist assumption (in which some folding of very high-frequency information onto lower frequency points occurs) and rounding errors inherent in any digital implementation of a mathematical transformation. However, these small errors, which will be most prevalent at higher spatial frequencies, cannot account either for the massive failure of the spectral representation to fit the experimental data or for the fact that the fit gets better when the high-frequency portions of the spectra are included. Finally, there is a possibility that the experimental data, determined after all with flashed stimulus presentation, is primarily the result of responses of transient channels, and not the sustained channels of our spectra model. However, again this is inconsistent with the result that the fit to the data improves when the high spatial frequency components are included, since it is known that transient channels respond best to low spatial frequency stimulation (Kulikowski \& Tolhurst, 1973).

Again, the best predictions of experimental con- 
fusability are template methods of the raw lettersan argument for a feature analytic perceptual process. That is, physically similar features in similar spatial position will create better template matches between letters. Overlap of features will produce both higher correlations between letters and also more similar feature decompositions of the experimentally presented letter stimuli, leading to greater confusability.

In general, the best predictions of experimental confusability among the spectra were produced by the most detailed spectra: the raw Fourier transforms with all frequency bins included. Since it appears that the visual system cannot maintain this level of detail in its spectral decomposition (Sachs, et al., 1971; Stromeyer \& Julesz, 1972), the probable spectral information available to the brain is more like the shaped octave-wide bands we calculated. Since the results for this set of spectra is considerably poorer than the results for the detailed, complete spectra, this is another argument against the use of spatial frequency information in perception of the letters.

On the other hand, stimuli like letters which are commonly seen in many different shapes (typefaces, different handwriting styles, etc.) may be better processed by a feature approach than stimuli which are not so well learned. Association of confusability of nonsense forms with their spectral similarity may give a better match than do the letters.

While much data has tended to support a spatial frequency coding model, much also seems inconsistent with it (Sekuler, 1974). Apparently, a model such as outlined above cannot be solely responsible for perceptual performance, even with near threshold stimuli. Nevertheless, some spatial frequency coding may coexist with feature analytic processes (Weisstein, 1973), and whichever mechanism is most useful for a particular task is used by the observer. Pooling of receptive field output into size-tuned channels does not rule out parallel pooling of output into feature channels. However, a two-dimensional spatial frequency analysis is a complex pattern in its own right, and if high-level pattern recognition processes must work on it, little is gained by its presence over doing the pattern recognition on the original input stimuli. In fact, the present results show that probably the original stimuli do better than the spectral analyses.

\section{REFERENCE NOTE}

1. Gibson, E. J., Osser, H., Shiff, W., \& Smith. J. An analysis of critical features of letters, tested by a confusion matrix. In $\mathrm{H}$. Levin (Ed.), $A$ basic research program on reading. Cornell Research Paper No. 639, Cornell University, 1963.

\section{REFERENCES}

Campbell, F. W., \& Robson, J. G. Application of Fourier analysis to the visibility of gratings. Journal of Physiology (London), 1968, 197, 551-566.

Carlson, C. R., Cohen, R. W., \& Gorog, I. Visual processing of simple two-dimensional sine-wave luminance gratings. Vision Research, 1977, 17, 351-358.

Fisher, D. F., Monty, R. A., \& Guucksburg, S. Visual confusion matrices: Fact or artifact? Journal of Psychology, 1969, 71, 111-125.

Giвson, E. J. Principles of perceptual learning and development. New York: Appleton-Century-Crofts, 1969.

GoBle, L. G. Filtered 2-dimensional discrete Fourier and Walsh transform correlation with recognition errors and similarity judgements. PhD dissertation, University of Michigan, 1975.

GraHAM, N. Spatial-frequency channels in human vision: Detecting edges without edge detectors. In C. S. Harris (Ed.), Visual coding and adaptibility. Hillsdale, N.J: Erlbaum, 1976.

GRAHAM, N., \& Nachmias, J. Detection of grating patterns containing two spatial frequencies: A comparison of singlechannel and multiple-channel models. Vision Research, 1971. 11, 251-259.

Granlund, G. H. Fourier preprocessing for hand print character recognition. IEEE Transactions on Computers, February 1972, 195-201.

Henning. G. B., Hertz, B. G., \& Broadbent, D. E. Some experiments bearing on the hypothesis that the visual system analyzes spatial patterns in independent bands of spatial frequency. Vision Research, 1975, 15, 887-897.

HoDGE, D. C. Legibility of a uniform-strokewidth alphabet: I. Relative legibility of upper and lower case letters. Journal of Engineering Psychology. 1962, 1, 34-46.

Kabrisky, M., Tallman, O., Day, C. M., \& Radoy, C. M. A theory of pattern perception based on human physiology. Ergonomics, 1970, 13, 129-147.

Kulikowski, J. J., \& Tolhurst, D. J. Psychophysical evidence for sustained and transient detectors in human vision. Journal of Physiology, 1973, 232, 149-162.

KüNNAPAS, T. Visual perception of capital letters. Scandinavian Journal of Psychology, 1966, 7, 189-196.

Maffei, L., \& Fiorentini. A. The visual cortex as a spatial frequency analyzer. Vision Research, 1973, 13, 1255-1267.

NeIsser, U. Cognitive psychology. New York: Appleton-CenturyCrofts, 1967.

Pollen. D. A.. TAylor, J. H., \& Lee, J. R. Does the striate cortex begin reconstruction of the visual world? Science, 1972, 176, 316-319.

Quick. R. F., \& Reichert, T. A. Spatial frequency selectivity in contrast detection. Vision Research, 1975, 15, 637-643.

Sachs, M. B., Nachmias, J., \& Robson, J. G. Spatial frequency channels in human vision. Journal of the Optical Society of America, 1971, 61, 1176-1186.

Sekuler, R. Spatial vision. Annual Review of Psychology, 1974, 25, 195-232.

Selfridge, O. G. Pandemonium: A paradigm for learning. In The mechanisation of thought processes. London: H. M. Stationery Office, 1959.

Stromeyer, C. F., \& Julesz, B. Spatial-frequency masking in vision: Critical bands and spread of masking. Journal of the Optical Society of America, 1972, 62, 1221-1232.

Thomas, J. P. Model of the function of receptive fields in human vision. Psychological Review, 1970, 77, 121-134.

TownSEnd, J. T. Theoretical analysis of an alphabetic confusion matrix. Perception \& Psychophysics, 1971, 9, 40-50.

WEBB, C. Practical use of the fast Fourier transform algorithm in time series analysis. NAT, National Technical Information Service Publication AD 713-166, 1970.

Weisstein, N. Beyond the yellow-Volkswagen detector and the grandmother cell: A general strategy for the exploration of operations in human pattern recognition. In R. L. Solso (Ed.), Contemporary issues in cognitive psychology: The Loyola Symposium. New York: Wiley, 1973. Pp. 17-51. 